\title{
Pseudokyste pancréatique compressif
}

\section{Pancreatic Pseudocyst Compression}

\section{A. Lefevre-Scelles • A. Marchalot • M. Antonietti}

Reçu le 27 août 2014; accepté le 5 novembre 2014

(C) SFMU et Lavoisier SAS 2014
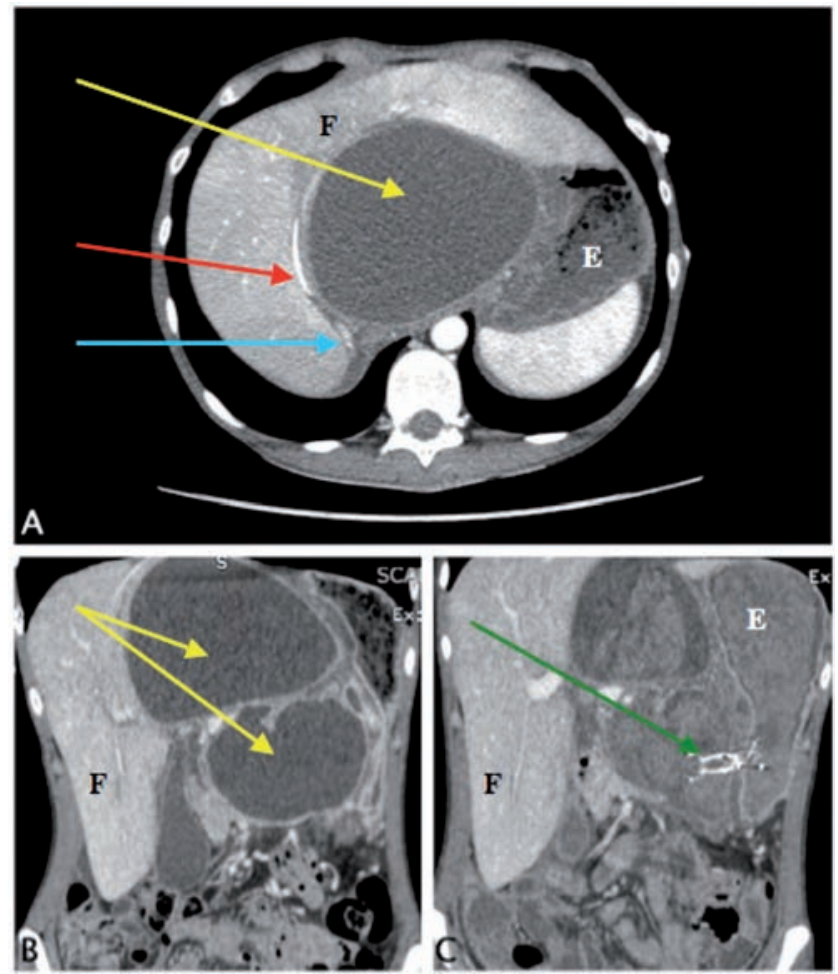

Fig. 1 A, B : tomodensitométrie abdominale injectée permettant de mettre en évidence un volumineux pseudokyste pancréatique bilobé (flèche jaune) comprimant à la fois la veine cave inférieure (flèche bleue) et les vaisseaux hépatiques (flèche rouge). (C) Tomodensitométrie abdominale injectée à $48 \mathrm{~h}$ de l'endoscopie digestive témoignant de la décompression partielle des organes adjacents après le drainage et la mise en place de la prothèse kystogastrique sous endoscopie (flèche verte). F : Foie ; E : Estomac

A. Lefevre-Scelles $(\bowtie) \cdot$ A. Marchalot

Réanimation médicale, CHU Charles Nicolle, Rouen, France

e-mail : antoine.lefevre-scelles@chu-rouen.fr

M. Antonietti

Gastroentérologie, CHU Charles Nicolle, Rouen, France
Une femme de 27 se présente aux urgences pour un tableau de douleurs épigastriques récurrentes associées à une altération de l'état général depuis cinq mois. L'interrogatoire de la patiente permet de noter une intoxication éthylique chronique (supérieure à $200 \mathrm{~g}$ par jour) depuis plus d'un an. La présence d'une lipasémie élevée (550 UI/l) a fait réaliser une tomodensitométrie abdominale injectée rapidement après l'admission aux urgences. L'examen a permis de mettre en évidence un volumineux pseudokyste pancréatique bilobé responsable d'une compression importante des vaisseaux hépatiques, de la veine cave inférieure, du foie et de l'estomac (Fig. 1 A,B). L'ensemble de ces éléments permet de confirmer le diagnostic de pancréatite aiguë sur pancréatite chronique alcoolique. La patiente a bénéficié d'une endoscopie gastroduodénale sous anesthésie générale afin de réaliser un drainage du kyste [1] et de mettre en place une prothèse kystogastrique (Fig. 1 C). Une nouvelle tomodensitométrie réalisée $48 \mathrm{~h}$ après la procédure permet d'objectiver le drainage partiel du kyste malgré la bonne position de la prothèse (Fig. $1 \mathrm{C}$ ). Soixante-douze heures après le geste endoscopique, la patiente a présenté un sepsis sévère compliqué d'une défaillance hémodynamique et respiratoire. Elle a rapidement été admise dans notre unité de réanimation médicale pour la prise en charge d'un choc septique sur un probable épisode de translocation bactérienne. L'évolution a été rapidement favorable sous antibiothérapie adaptée, la patiente est sortie de réanimation médicale deux jours plus tard.

\section{Référence}

1. Dítě P, Novotný I, Lata J, et al (2014) Endoscopic drainage treatment of pancreatic pseudocysts. Hepatogastroenterology 61:497 